J. DIFFERENTIAL GEOMETRY

$\mathbf{7 7}$ (2007) 169-173

\title{
ERRATUM: A LOCAL PROOF OF PETRI'S CONJECTURE AT THE GENERAL CURVE
}

\author{
Herb Clemens
}

\begin{abstract}
A generation of symbols asserted for $n \geq 0$ in the proof of Theorem 3.3 of the original paper in fact only holds for $n>0$, thus undermining the proof of the theorem. A new version of Section 3.5 of the original paper is given, culminating in a corrected proof of Theorem 3.3. The author thanks Deepak Khosla for pointing out the gap in the previous version of the proof.
\end{abstract}

\subsection{Extendable linear systems on curves.}

If $M$ denotes a sufficiently small analytic neighborhood of a general point in the moduli space of curves of genus $g$, with universal curve $C / M$, there is a stratification of the locus

$$
Z_{d}^{r}=\left\{L: L \text { globally generated, } h^{0}(L)=r+1\right\} \subseteq \operatorname{Pic}^{d}(C / M)
$$

such that all strata are smooth and the projection of each to $M$ is submersive with diffeomeorphic fibers. Next consider the induced stratification of the pre-image of $Z_{d}^{r}$ under the Abel-Jacobi map

$$
\alpha: C^{(d)} / M \rightarrow \operatorname{Pic}^{d}(C / M) .
$$

By considering the contact locus between this pre-image stratification and the various diagonal loci in $C^{(d)} / M$, one can construct a refinement of the stratification of

$$
\alpha^{-1}\left(Z_{d}^{r}\right) \subseteq C^{(d)} / M
$$

such that all strata are smooth and the projection of each to $M$ is submersive with diffeomorphic fibers and having the additional property that, beginning with the initial element $(d)$ of the partially ordered set $\left\{\left(d_{1}, \ldots, d_{s}\right)\right\}$ of all partitions of $d$, the stratification is compatible with each set

$$
\operatorname{diag}_{\left(d_{1}, \ldots, d_{s}\right)}\left(C^{(d)} / M_{g}\right) \cap \alpha^{-1}\left(Z_{d}^{r}\right) .
$$

Suppose now that $C_{0}$ is a compact Riemann surface of genus $g$ of general moduli and that $L_{0}$ is a line bundle of degree $d$ on $C_{0}$ such that the linear system $\mathbb{P}_{0}:=\mathbb{P}\left(H^{0}\left(L_{0}\right)\right)$ is basepoint-free. Let $C_{\beta} / \Delta$ be a Schiffer variation supported at a finite set $A_{0} \subseteq C_{0}$. Then, by genericity

Received May, 2006. 
of $C_{0}$ and the remarks just above, there is a deformation $\mathbb{P}_{\Delta} \subseteq C_{\beta}^{(d)}$ over $\Delta$ of $\mathbb{P}_{0} \subseteq C_{0}^{(d)}$ for which there exists a trivialization

$$
T: \mathbb{P}_{\Delta} \rightarrow \mathbb{P}_{0} \times \Delta
$$

compatible with each partition locus of $d$, that is, for each partition $\left(d_{1}, \ldots, d_{s}\right)$ of $d$,

$$
\begin{aligned}
& T\left(\operatorname{diag}_{\left(d_{1}, \ldots, d_{s}\right)}\left(C_{\beta}^{(d)}\right) \times_{C_{\beta}^{(d)}} \mathbb{P}_{\Delta}\right) \\
& =\left(\operatorname{diag}\left(d_{1}, \ldots, d_{s}\right)\right. \\
& \left.\left(C_{0}^{(d)}\right) \times_{C_{0}^{(d)}} \mathbb{P}_{0}\right) \times \Delta .
\end{aligned}
$$

Notice that $T$ is a $C^{\infty}$-map, and is not in general analytic. However $T$ can be chosen so that, for each $p \in \mathbb{P}_{0}, T^{-1}(\{p\} \times \Delta)$ is a proper analytic subvariety of $\mathbb{P}_{\Delta}$.

Now the tautological section $\tilde{f}_{0}$ of $\tilde{L}_{0}(1)=\mathcal{O}_{\mathbb{P}_{0}} \otimes L_{0}$ defined in $(27)$ has divisor

$$
D_{0} \subseteq \mathbb{P}_{0} \times C_{0}
$$

Let

$$
D \subseteq \mathbb{P}_{\Delta} \times{ }_{\Delta} C_{\beta}
$$

denote the divisor of the tautological section $\tilde{f}$ of

$$
\tilde{L}(1):=\mathcal{O}_{\mathbb{P}_{\Delta}}(1) \otimes_{\Delta} L .
$$

Then, by (31), the "product" trivialization

$$
\left(T, F_{\beta}\right): \mathbb{P}_{\Delta} \times{ }_{\Delta} C_{\beta} \rightarrow \mathbb{P}_{0} \times C_{0} \times \Delta
$$

is compatible with the trivialization $T$ in (30), that is, for each $p \in \mathbb{P}_{0}$,

$$
\left(T, F_{\beta}\right)^{-1}\left(\{p\} \times C_{0} \times \Delta\right)=T^{-1}(\{p\} \times \Delta) \times_{\mathbb{P}_{\Delta}}\left(\mathbb{P}_{\Delta} \times \times_{\Delta} C_{\beta}\right) .
$$

That is, we have the commutative diagram

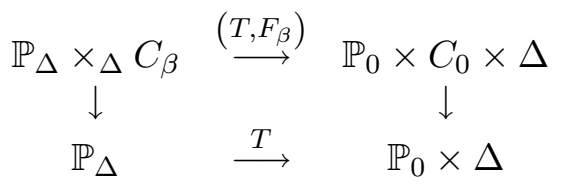

Furthermore, by $(31)$, we can adjust $\left(T, F_{\beta}\right)$ "in the $C_{0}$-direction" to obtain a trivialization

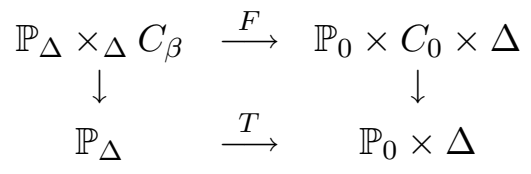

which maintains the property

$$
F^{-1}\left(\{p\} \times C_{0} \times \Delta\right)=T^{-1}(\{p\} \times \Delta) \times_{\mathbb{P}_{\Delta}}\left(\mathbb{P}_{\Delta} \times{ }_{\Delta} C_{\beta}\right) .
$$

and achieves in addition that

$$
F^{-1}\left(D_{0} \times \Delta\right)=D \text {. }
$$


Finally, we can choose the adjustments to be holomorphic in the $C_{0^{-}}$ direction in a small neighborhood of $\mathbb{P}_{\Delta} \times A_{0} \times \Delta$.

Thus referring to Lemma 2.7 there is a $C^{\infty}$-vector field

$$
\gamma=\sum_{n>0} \gamma_{n} t^{n}
$$

on $\mathbb{P}_{0} \times C_{0} \times \Delta$ of type $(1,0)$ such that

1) each $\gamma_{n}$ annihilates functions pulled back from $\mathbb{P}_{0}$, that is, it is an $\mathcal{O}_{\mathbb{P}_{0}}$-linear operator,

2) for each $n$ and each $p \in \mathbb{P}_{0}$,

$$
\left.\gamma_{n}\right|_{\{p\} \times C_{0}}
$$

is meromorphic on a neighborhood of $\{p\} \times A_{0}$,

3) given a function

$$
g=\sum_{k=0}^{\infty} g_{k} t^{k}: \mathbb{P}_{0} \times C_{0} \times \Delta \rightarrow \mathbb{C}
$$

with each $g_{k}$ a $C^{\infty}$-function on (an open set in) $\mathbb{P}_{0} \times C_{0}$ and any point $p \in \mathbb{P}_{0}$,

$$
\left.g \circ F\right|_{T^{-1}(\{p\} \times \Delta) \times_{\mathbb{P}_{\Delta}}}\left(\mathbb{P}_{\Delta} \times{ }_{\Delta} C_{\beta}\right)
$$

is holomorphic if and only if

$$
\left.\left[\overline{\partial_{0}}, e^{L_{-\gamma}}\right](g)\right|_{\{p\} \times C_{0} \times \Delta}=0 .
$$

Again, following Lemma 3.2, there is a trivalization

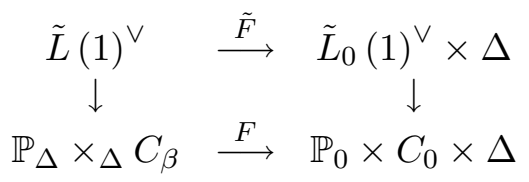

of $\tilde{L}(1)$ and a lifting $\tilde{\gamma}$ of $\gamma$ such that, for the tautological sections $\tilde{f}_{0}$ and $\tilde{f}$ defined earlier in this section,

$$
\tilde{f}=\tilde{F} \circ \tilde{f}_{0} .
$$

Thus, for each $p \in \mathbb{P}_{0}$,

$$
\left.\left[\overline{\partial_{0}}, e^{L_{-\tilde{\gamma}}}\right]\left(\tilde{f}_{0}\right)\right|_{\{p\} \times C_{0} \times \Delta}=0 .
$$

Let

$$
\mathfrak{D}_{n}^{\mathbb{P}_{0}}\left(\tilde{L}_{0}(1)\right) \subseteq \mathfrak{D}_{n}\left(\tilde{L}_{0}(1)\right)
$$

denotes the subsheaf of $\mathcal{O}_{\mathbb{P}_{0}}$-linear operators. Then

$$
\left[\overline{\partial_{0}}, e^{L_{-\tilde{\gamma}}}\right]
$$

is a $\overline{\partial_{0}}$-closed element of

$$
\sum_{n>0}^{\infty} H^{1}\left(\mathfrak{D}_{n}^{\mathbb{P}_{0}}\left(\tilde{L}_{0}(1)\right)\right) t^{n} .
$$


Now, referring to (29), we need to analyze

$$
\begin{aligned}
& \rho_{*}\left[\overline{\partial_{0}}, e^{L_{-}}\right] \in \sum_{n>0} H^{1}\left(\mathfrak{D}_{n}^{\prime}\right) t^{n} \\
& =\sum_{n>0} H^{1}\left(\mathfrak{D}_{n}\left(L_{0}\right)\right) \otimes \operatorname{End}\left(H^{0}\left(L_{0}\right)\right) t^{n} .
\end{aligned}
$$

In fact, by construction, this element lies in the image of

$$
\begin{aligned}
& \sum_{n>0}^{\infty} H^{1}\left(\rho_{*} \mathfrak{D}_{n}^{\mathbb{P}_{0}}(\tilde{L}(1))\right) t^{n} \\
& =\sum_{n>0} H^{1}\left(\mathfrak{D}_{n}\left(L_{0}\right)\right) \otimes \mathbb{C} \cdot(i d) \cdot t^{n} \\
& \subseteq \sum_{n>0} H^{1}\left(\mathfrak{D}_{n}\left(L_{0}\right)\right) \otimes \text { End }\left(H^{0}\left(L_{0}\right)\right) t^{n} .
\end{aligned}
$$

Now

$$
H^{1}\left(\tilde{L}_{0}(1)\right)=\operatorname{Hom}\left(H^{0}\left(L_{0}\right), H^{1}\left(L_{0}\right)\right) .
$$

But by (34), the image of

$$
\left.\left\{\left[\overline{\partial_{0}}, e^{L_{-\tilde{\gamma}}}\right]\left(\tilde{f}_{0}\right)\right\}\right|_{\{p\} \times C_{0} \times \Delta} \in \sum_{n>0}^{\infty} H^{1}\left(L_{0}\right) \cdot t^{n} .
$$

is zero for each $p \in \mathbb{P}_{0}$. Thus

$$
\rho_{*}\left[\overline{\partial_{0}}, e^{L_{-}}\right]\left(\rho_{*} \tilde{f}_{0}\right)=0 \in \sum_{n>0} \operatorname{Hom}\left(H^{0}\left(L_{0}\right), H^{1}\left(L_{0}\right)\right) t^{n} .
$$

Theorem 3.3. Suppose $X_{0}$ is a curve of genus $g$ of general moduli. Suppose further that, by varying the choice of $\beta$ in the Schiffer-type deformation in Section 3.3, the coefficients to $t^{n+1}$ in all expressions

$$
\left[\bar{\partial}, e^{-L_{\beta}}\right]
$$

generate $H^{1}\left(S^{n+1}\left(T_{X_{0}}\right)\right)$ for each $n \geq 0$. (For example we allow the divisor $A_{0} \subseteq X_{0}$ to move.) Then the maps

$$
\mu^{n+1}: H^{1}\left(S^{n+1} T_{X_{0}}\right) \rightarrow \frac{\operatorname{Hom}\left(H^{0}\left(L_{0}\right), H^{1}\left(L_{0}\right)\right)}{\text { image } \tilde{\mu}^{n}}
$$

are zero for all $n \geq 0$.

Proof. Let

$$
\rho_{*}\left[\overline{\partial_{0}}, e^{L_{-\tilde{\gamma}}}\right]_{n+1} .
$$

denote the coefficient of $t^{n+1}$ in $\rho_{*}\left[\overline{\partial_{0}}, e^{L_{-}} \tilde{\gamma}\right.$. Referring to (29) and the fact the the operators take values in the sheaf $\mathfrak{D}_{n}^{\mathbb{P}_{0}}\left(\tilde{L}_{0}(1)\right)$, we have that

$$
\begin{aligned}
& \text { symbol }\left(\left(\rho_{*}\left[\overline{\partial_{0}}, e^{L_{-\tilde{\gamma}}}\right]\right)_{n+1}\right) \\
& =\left(\bar{\partial} \beta_{1}^{n+1} \otimes 1\right) \oplus 0 \in S^{n+1}\left(T_{X_{0}}\right) \oplus\left(S^{n}\left(T_{X_{0}}\right) \otimes \operatorname{End}^{0}\left(H^{0}\left(L_{0}\right)\right)\right)
\end{aligned}
$$


where

$$
\beta=\sum_{j>0} \beta_{j} t^{j}
$$

By (36) and the hypothesis that the elements $\bar{\partial} \beta_{1}^{n+1}$ generate $H^{1}\left(S^{n+1} T_{X_{0}}\right)$, we have that, by varying $\beta$, the elements

$$
\operatorname{symbol}\left(\rho_{*}\left[\overline{\partial_{0}}, e^{L_{-\tilde{\gamma}}}\right]_{n+1}\right)
$$

generate

$$
S^{n+1}\left(T_{X_{0}}\right)
$$

for each $n \geq 0$.

Thus, by (29) and (35), the map $\tilde{\nu}^{n+1}$ given by

$$
\begin{aligned}
H^{1}\left(\mathfrak{D}_{n+1}\left(L_{0}\right)\right) & \rightarrow \frac{\operatorname{Hom}\left(H^{0}\left(L_{0}\right), H^{1}\left(L_{0}\right)\right)}{\operatorname{image}\left(\tilde{\nu}^{n}\right)} \\
D & \mapsto D\left(\tilde{f}_{0}\right)
\end{aligned}
$$

is zero for all $n \geq 0$.

q.e.d.

Current address: Mathematics Department Ohio State University Columbus, OH 43210

E-mail address: clemens@math.ohio-state.edu 